\title{
Joint Planning of Transmission and Distribution Network Considering Wind-solar Complementarity
}

\author{
Jie Yang ${ }^{1 *}$, Yaxin $\mathrm{Li}^{1}$, Dongjun Yang ${ }^{1}$, Hongsheng Zhao ${ }^{1}$, and Hanping $\mathrm{Xu}^{1}$ \\ ${ }^{1}$ state grid hubei electric power company limited economic research institute, wuhan, Hubei province, 430074, China
}

\begin{abstract}
The temporal and spatial distribution characteristics of wind power and photovoltaic output are complementary to a certain extent, which can stabilize the volatility of single new energy power generation to a certain extent and improve the ability of the grid to absorb new energy. This paper aims to study the joint planning method of power transmission and distribution network considering the complementary characteristics of wind-solar time and space. First, a wind-solar joint distribution model based on the timevarying Copula theory is established, and a wind-solar output simulation method considering the complementary characteristics of time and space is proposed. Then, taking the system investment and new energy consumption capacity as the optimization goals, and taking the system power balance, line transmission capacity, conventional generator set output and climbing capacity as constraints, the joint planning model of transmission and distribution network is established. At last, the analysis of calculation examples proves that the joint planning model of transmission and distribution network considering the complementary characteristics of wind-solar time and space can significantly improve the capacity of the grid to absorb new energy.
\end{abstract}

\section{Introduction}

As the proportion of new energy sources in the power structure continues to increase, its impact on the safe and stable operation of the power system has become increasingly significant. The contradiction between the development of new energy and the safe operation of the system is gradually emerging in some areas. The phenomenon of abandoned new energy power continues to emerge. The problem of new energy consumption has become one of the key issues that need to be considered in power grid planning, construction and operation.

Since the temporal and spatial distribution of wind power and photovoltaic power output is complementary to a certain extent, it can smooth the volatility of a single new energy generation. Therefore, the combined operation of wind-solar is beneficial to improve the ability of the grid to absorb new energy. Literature [1] constructed a grid expansion planning model for multiobjective wind farm access, but only considered the uncertain factor of wind power output. Literature [2] used the inverse Nataf transform to establish a wind speed correlation model, and build a multi-objective grid optimization planning model with wind farms considering the wind speed correlation. Literature [3] proposed a probability-based grid expansion planning model on the basis of considering the flexibility and uncertainty of wind power generation, in order to balance the economics of grid investment and demand response expenditures, and verify the effectiveness and reliability of the model. Literature [4-6] described the relevance of wind power based on the Copula theory, and proposed a source network planning method with the goal of optimal economy, without considering the complementary output of wind farms and photovoltaic power plants. However, in the future power grid planning, a high proportion of new energy access has become inevitable, and it is necessary to consider the correlation between wind farms and photovoltaic power plants when conducting grid planning.

This paper aims to study the joint planning method of transmission and distribution network considering the complementary characteristics of wind-solar time and space. First, a wind-solar joint distribution model based on the time-varying Copula theory is established, and a wind-solar output simulation method considering the complementary characteristics of time and space is proposed. Then, taking the system investment and new energy consumption capacity as the optimization goals, and taking the system power balance, line transmission capacity, conventional generator set output and climbing capacity as constraints, the joint planning model of transmission and distribution network is established. At last, the analysis of calculation examples proves that the joint planning model of transmission and distribution network considering the complementary characteristics of wind-solar time and space can significantly improve the capacity of the grid to absorb new energy. 


\section{Wind-solar joint distribution model based on time-varying copula theory}

The correlation between two time-varying related variables is described by the bivariate normal Copula model, and the parameters of this model change with time. The marginal distribution of the variables themselves can be described by choosing the distribution form. The Copula function part of the correlation between variables is expressed as follows.

$$
\begin{aligned}
& C\left(u, v ; \rho_{N, t}\right)=\int_{-\infty}^{\Phi^{-1}(u)} \int_{-\infty}^{\Phi^{-1}(v)}\left[\frac{1}{2 \pi \sqrt{1-\rho_{N, t}^{2}}} \mathrm{~g}\right. \\
& \exp \left(-\frac{s^{2}-2 \rho_{N, t} s r+r^{2}}{2\left(1-\rho_{N, t}^{2}\right)}\right) d s d r
\end{aligned}
$$

Where $s, r$ are related variables of the distribution function, $\Phi-1($.$) is inverse function of standard normal$ distribution, $\rho \mathrm{N}, \mathrm{t}$ is correlation coefficient and its timevarying equation is,

$$
\begin{aligned}
& \rho_{N, t}=\Lambda\left[\omega_{N}+\beta_{N} \rho_{N, t}+\right. \\
& \left.\alpha_{N} \frac{1}{m} \sum_{j=1}^{m} \Phi^{-1}\left(u_{t-j}\right) \Phi^{-1}\left(v_{t-j}\right)\right]
\end{aligned}
$$

Where $\Lambda(\mathrm{x})$ is related variables of the logistic function, $\omega \mathrm{N}, \beta \mathrm{N}, \alpha \mathrm{N}$ are time-varying equation parameters, $\mathrm{m}$ is the data window, usually 10 .

The above parameters estimation method of the Copula function can adopt a two-stage maximum likelihood estimation method. The principle is that estimate the respective marginal distribution parameters of the variables firstly, and then use the marginal distribution parameters as known conditions to estimate the relevant parameters in the Copula function model.

\section{Wind-solar output simulation method considering the complementary characteristics of time and space}

In this section, a probability model to accurately describe the complementary characteristics of the regional windsolar power generation is established, using the strength of the complementary characteristics as the basis of the division model and using one year as the sampling period to conduct probability modeling, that is, classify January, February, March, April, September, and December as weak complementary characteristics season, classify May, June, July, August, October, and November as strong complementary characteristics season, and considering the difference in complementary characteristics at each moment of the two seasons. The parameters of the Plackett Copula function, which measure the strength of the complementary characteristics of each period of the day, are shown in Table 1 .
Table 1. Plackett Copula function parameters at various times during the day.

\begin{tabular}{|c|c|c|c|c|c|}
\hline $\begin{array}{c}\text { Time } \\
\text { period }\end{array}$ & $\begin{array}{c}\text { Strong } \\
\text { season }\end{array}$ & $\begin{array}{c}\text { Weak } \\
\text { season }\end{array}$ & $\begin{array}{c}\text { Time } \\
\text { period }\end{array}$ & $\begin{array}{c}\text { Strong } \\
\text { season }\end{array}$ & $\begin{array}{c}\text { Weak } \\
\text { season }\end{array}$ \\
\hline $\mathbf{7 - 8}$ & 0.6192 & $/$ & $\mathbf{1 3 - 1 4}$ & 0.4379 & 0.7094 \\
\hline $\mathbf{8 - 9}$ & 0.5605 & 0.9357 & $\mathbf{1 4 - 1 5}$ & 0.4526 & 0.7460 \\
\hline $\mathbf{9 - 1 0}$ & 0.5153 & 0.9004 & $\mathbf{1 5 - 1 6}$ & 0.5273 & 0.7952 \\
\hline $\mathbf{1 0 - 1 1}$ & 0.4735 & 0.8677 & $\mathbf{1 6 - 1 7}$ & 0.5800 & 0.7925 \\
\hline $\mathbf{1 1 - 1 2}$ & 0.4739 & 0.8195 & $\mathbf{1 7 - 1 8}$ & 0.6377 & 0.8952 \\
\hline $\mathbf{1 2 - 1 3}$ & 0.4207 & 0.7749 & $\mathbf{1 8 - 1 9}$ & 0.6324 & $/$ \\
\hline
\end{tabular}

The joint probability density function of daytime wind-solar power output is composed of the data independent distribution function and the Plackett Copula function considering complementary characteristics in the statistical time period. The dual probability distribution function is as follows.

$$
\begin{gathered}
F(x, y)=C\left(f_{1}(x), f_{2}(x), \theta\right) \\
C(u, v)=\frac{s-\sqrt{s^{2}-4 u v \theta(\theta-1)}}{2(\theta-1)} \\
s=1+(\theta-1)(u+v)
\end{gathered}
$$

Where $\mathrm{fl}(\mathrm{x})$ and $\mathrm{f} 2(\mathrm{x})$ are the marginal distribution functions of wind power and photovoltaic output, $\theta$ is the parameter of Plackett Copula function. The Monte Carlo method is used to extract power generation data based on its probability density function, which can be used to simulate the wind and daylight output within a year.

Photovoltaic power generation system does not generate power at night, thus the probability density function of wind power generation system output is its marginal probability density function. The Monte Carlo method is also used to extract power generation data based on its probability density function, and the actual situation of power generation is simulated to obtain wind power output. Finally, the wind power output data with time difference complementary characteristics is obtained by simulation.

In order to compare with the wind power and photovoltaic sampling data with complementary characteristics, the wind power and photovoltaic sampling data without complementary characteristics are obtained, according to the independent probability density function sample at each time using the Monte Carlo method.

Figures 1(a) and 1(b) are comparison diagrams of the simulated wind-solar sampling data with complementary characteristics and the wind-solar sampling data without complementary characteristics in the first week of January. It can be seen from the figure that after adding complementary characteristic sampling, the sampling result will be corrected, so that the output of wind power and photovoltaic power will be small or large at the same 
time. Therefore, it can be seen that the Monte Carlo sampling method correctly simulates the complementary

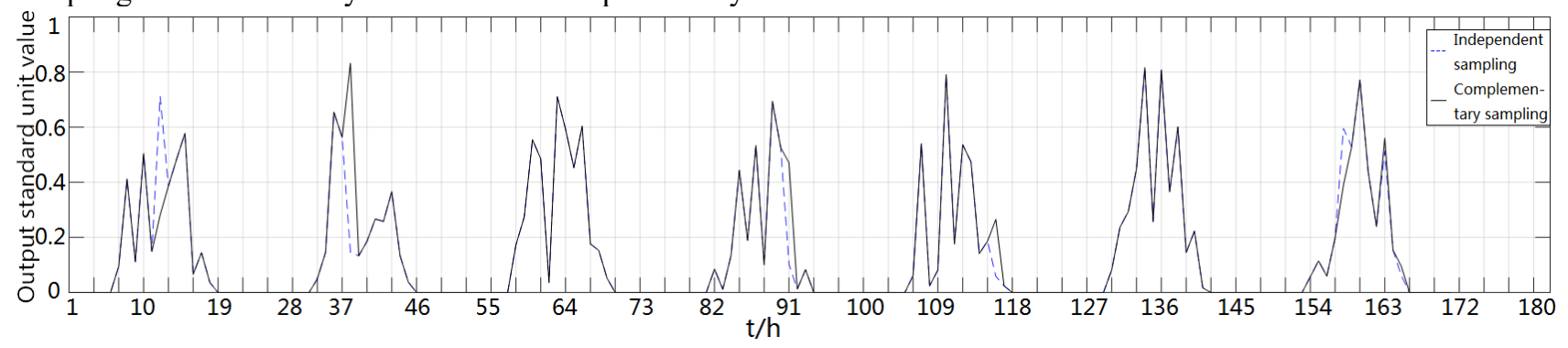

Figure 1(a). Output curves comparison of photovoltaic systems with and without complementary characteristics.

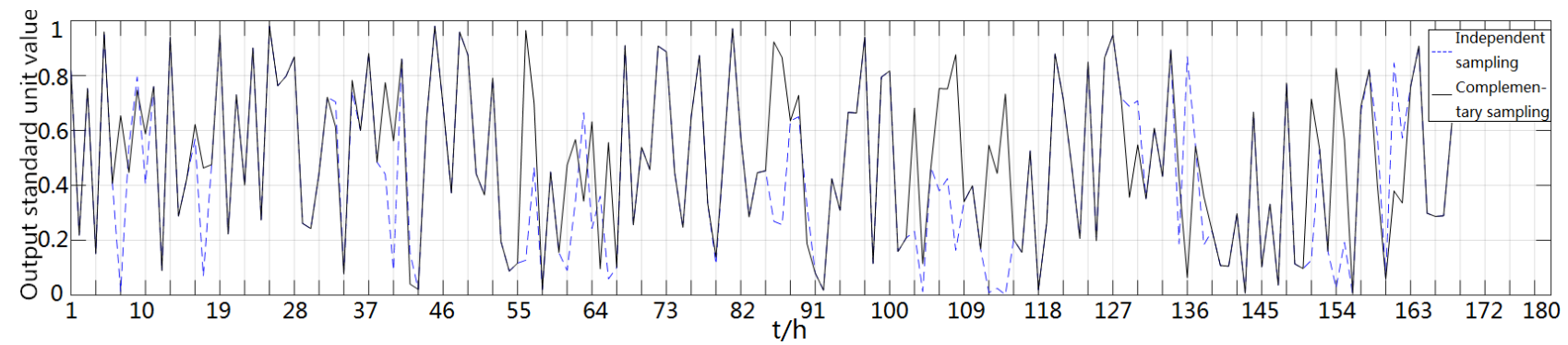

Figure 1(b). Output curves comparison of wind power systems with and without complementary characteristics.

\section{Transmission grid planning model}

This section constructs the transmission grid planning model, mainly considering the cost of centralized thermal power generation of the grid. The planning cost of the transmission grid is as follows.

$$
F_{1}=\sum_{t \in T} \sum_{i \in N}\left(C_{i, g} P_{i}+C_{i, o} P_{i, t}\right)
$$

Where $\mathrm{T}$ is the total number of periods in the planning cycle, $\mathrm{N}$ is power collection of transmission grid, $\mathrm{Ci}, \mathrm{g}$ is the fixed investment cost of the ith power supply, $\mathrm{Ci}, \mathrm{o}$ is the operating cost of the ith power supply, $\mathrm{Pi}$ is the rated capacity of the ith power supply, $\mathrm{Pi}, \mathrm{t}$ is the real-time power generation of the ith power supply.

The constraints of transmission grid planning mainly include:

a. power balance constraint

$$
\sum_{i \in N} P_{i, t}=\sum_{\mathrm{k}=\mathrm{n}} P_{B u y, t}^{k}+\sum_{r \in \mathrm{D}} P_{r, t}+\sum_{l \in N_{L}} P_{l o s s, l, t}
$$

Where $\mathrm{N}$ is the power collection of transmission grid, $\mathrm{n}$ is the set of distribution networks connected to the transmission grid, D is the load nodes set of the transmission grid, NL is the branches set of the transmission grid, PkBuyt is the power transmitted from the transmission grid to the kth distribution network during the period $t, \mathrm{Pr}, \mathrm{t}$ is the load power of the load node $r$ of the transmission grid in the period $t$, and Ploss, $1, t$ is the loss of the lth branch of the transmission network in the period $t$.

b. line transmission power constraint

$$
P_{l, t} \leq P_{l, \max }
$$

Where $\mathrm{Pl}, \mathrm{t}$ is the transmission power of the power transmission branch 1 in the time period $\mathrm{t}$, and $\mathrm{Pl}$, max is the maximum allowable transmission power of the power transmission branch 1 .

c. conventional generator set output constraints

$$
P_{i, \min } \leq P_{i, t} \leq P_{i, \max }
$$

Where $P_{i, \text { min }}$ is the minimum allowable power generation of the $i^{\text {th }}$ power supply, and $P_{i, \max }$ is the maximum allowable power generation of the $i^{\text {th }}$ power supply.

d.conventional generator set climbing power constraint

$$
\left\{\begin{array}{l}
P_{i, t}-P_{i, t-1} \leq R_{u i} \\
P_{i, t-1}-P_{i, t} \leq R_{d i}
\end{array}\right.
$$

Where $R_{d i}$ is the minimum downhill rate, and $R_{u i}$ is the maximum uphill rate.

\section{Distribution network planning model}

The planning model of the distribution network mainly considers its investment cost and operating cost. This paper does not consider new lines and distribution network reconstruction, so the investment cost is mainly the investment cost of DG. The operating cost of distributed new energy generation is very small, and this paper does not consider it, so the operating cost is the cost of network loss. 


$$
\left\{\begin{array}{l}
F_{2, k}=C_{\text {loss }}^{k}+C_{D G}^{k} \\
C_{D G}^{k}=\sum_{m \in N_{k, D G}} c_{D G, m} P_{D G, m}^{k}
\end{array}\right.
$$

Where $F_{2, k}$ is the total cost of the $k^{\text {th }}$ distribution network, $C^{k}{ }_{D G}$ is the DG investment cost of the $k^{\text {th }}$ distribution network, $C^{k}$ loss is the loss cost of the $k^{\text {th }}$ distribution network, $N_{k, D G}$ is the DG nodes set of the $k^{\text {th }}$ distribution network, $C_{D G, m}$ is the $m^{\text {th }}$ DG initial investment cost of the $k^{\text {th }}$ distribution network, $P_{D G, m}^{k}$ is the $m^{\text {th }}$ DG capacity of the $k^{\text {th }}$ distribution network.

The internal constraints of distribution network planning mainly include the following.

a. power balance constraint

$$
P_{B u y, t}^{k}+\sum_{\mathrm{m} \in \mathrm{N}_{k, D G}} b_{m}(t) P_{D G, m}^{k}=\sum_{f \in N_{f}} P_{\mathrm{f}, \mathrm{t}}^{k}+P_{t}^{k}
$$

Where $b_{m}(t)$ is the actual output versus capacity curve of the $m^{\text {th }}$ DG of the $k^{\text {th }}$ distribution network, and $P_{t}^{k}$ is the initial power of each node of the $k^{\text {th }}$ distribution network.

b. node voltage amplitude constraint

$$
V_{i, \min }^{k} \leq V_{i}^{k} \leq V_{i, \text { max }}^{k}
$$

Where $V_{i}^{k}$ is the the $i^{\text {th }}$ node voltage of the $k^{\text {th }}$ distribution network, $V^{k}{ }_{i m i n}$ is the $i^{\text {th }}$ node minimum allowable voltage of the $k^{\text {th }}$ distribution network, and $V^{k}{ }^{k}$ max is the $i^{\text {th }}$ node maximum allowable voltage of the $k^{\text {th }}$ distribution network.

c. line capacity constraints

$$
P_{l, \text { min }}^{k} \leq P_{l, t}^{k} \leq P_{l, \text { max }}^{k}
$$

Where $P^{k}{ }_{l t}$ is the $l$ branch transmission power of the $k^{\text {th }}$ distribution network, $P^{k}{ }_{l \min }$ is the minimum allowable transmission power of the $l$ branch of the $k^{\text {th }}$ distribution network, $P^{k}{ }_{\max }$ is the maximum allowable transmission power of the $l$ branch of the $k^{\text {th }}$ distribution network.

d. DG capacity constraints

$$
\sum P_{m, D G}^{k} \leq \eta_{k} \sum P_{t}^{k}
$$

Where $\eta_{k}$ is the maximum penetration rate of DG in the $k^{\text {th }}$ distribution network, $\Sigma P^{k}{ }_{m D G}$ is the sum of the capacity of DG in the $k^{\text {th }}$ distribution network, and $\Sigma P^{k}{ }_{t}$ is the sum of active power of all nodes in the $k^{\text {th }}$ distribution network.

\section{Joint planning model of transmission and distribution network}

The objective function of the joint planning model of the transmission and distribution network is the sum of the investment and operation cost of the transmission network and the distribution network.

$$
F=F_{1}+\sum_{k=1}^{K} F_{2, k}
$$

Where $F$ is the total cost of grid planning, $F_{1}$ is the investment and operation cost of the transmission grid, $F_{2 k}$ is the total cost of the $k^{\text {th }}$ distribution network, and $K$ is the number of distribution networks participating in the planning.

In addition to the constraints of transmission grid planning and distribution network planning, the internal constraints of power transmission and distribution network planning should also include wind and solar complementary capacity constraints to ensure that the proportion of wind and solar power generation systems is within a certain range and that the complementary characteristics can be reflected.

$$
a \leq \frac{\sum P_{\text {wind }}^{k}}{\sum P_{\text {Solar }}^{k}} \leq b
$$

Where $a$ is the lower limit of the proportion of windsolar power generation systems, $b$ is the upper limit of the proportion of wind-solar power generation systems, $\Sigma P^{k}{ }_{\text {wind }}$ is the total capacity of the $k^{\text {th }}$ distribution network wind power system, $\Sigma P^{k}$ solar is the total capacity of the photovoltaic power generation system, and $\Sigma P^{k}$ wind is the total capacity of the wind power system.

In addition to wind-solar hybrid capacity constraints, the constraints of this model also include transmission grid constraints and distribution network constraints.

\section{Calculation example of joint planning of transmission and distribution network}

The case for analysis in this paper is shown in figure 2. The transmission grid selects the IEEE 9-node power transmission system, and the distribution network selects the IEEE 33-node power distribution system and the IEEE 69-node power distribution system respectively. WTG is a distributed wind power station, and PVG is a distributed photovoltaic power station, which are connected to different nodes of the two power distribution network systems. 


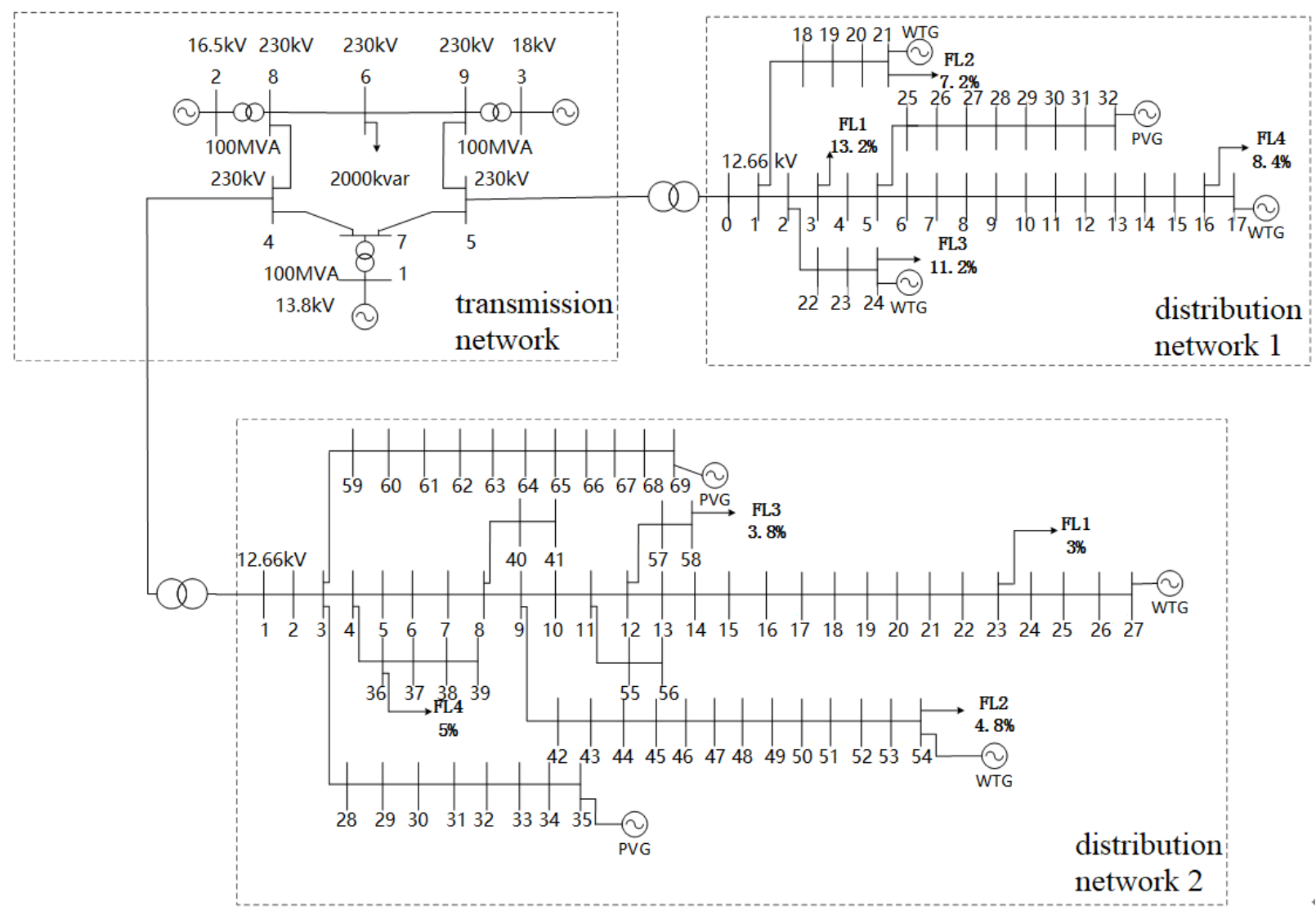

Figure 2. Calculation model of joint planning of transmission and distribution network.

The raw data of each IEEE node comes from the standard IEEE model. Data such as power investment and operating cost, and time-of-use electricity price of the distribution network are shown in Table 2 and Table 3 .

Table 2. Power investment and operating costs.

\begin{tabular}{|c|c|c|}
\hline $\begin{array}{c}\text { Power } \\
\text { generation } \\
\text { type }\end{array}$ & $\begin{array}{c}\text { Fixed investment } \\
\text { expenses } \\
(\mathbf{1 0} \text { yuan/kW) }\end{array}$ & $\begin{array}{c}\text { Operation and } \\
\text { maintenance costs } \\
\text { (yuan/kWh) }\end{array}$ \\
\hline $\begin{array}{c}\text { Distributed } \\
\text { wind power }\end{array}$ & 0.3 & 0 \\
\hline $\begin{array}{c}\text { Distributed } \\
\text { photovoltaic } \\
\text { power }\end{array}$ & 0.5 & 0 \\
\hline $\begin{array}{c}\text { Centralized } \\
\text { thermal } \\
\text { power }\end{array}$ & 0.5768 & 0.568 \\
\hline
\end{tabular}

Table 3. Time-of-use electricity price for distribution network.

\begin{tabular}{|c|c|c|}
\hline Time & $7: 00-21: 00$ & $22: 00-6: 00$ \\
\hline $\begin{array}{c}\text { Price } \\
\text { (yuan/kWh) }\end{array}$ & 0.7511 & 0.3755 \\
\hline
\end{tabular}

The two sets of wind-solar output are respectively used as power input into the planning model in Section 5, and the interior point method is used to solve the problem. Among them, wind 1, wind 2, wind 3, and solar 4 are the power supply numbers on the side of the distribution network 1 , and wind 5 , wind 6 , solar 7, and solar 8 are the power supply numbers on the side of the distribution network 2. The results of the two calculations are shown in Table 4.

Table 4. List of new energy installed capacity before and after considering the complementary characteristics of wind-solar.

\begin{tabular}{|c|c|c|}
\hline Capacity & $\begin{array}{c}\text { Independent } \\
\text { contribution }\end{array}$ & $\begin{array}{c}\text { Complementary } \\
\text { contributions }\end{array}$ \\
\hline Wind 1 & 207.16 & 90.48 \\
\hline Wind 2 & 0 & 83.27 \\
\hline Wind 3 & 404.7 & 895.61 \\
\hline Wind 4 & 0.05 & 66.34 \\
\hline Wind 5 & 0.01 & 10.34 \\
\hline Wind 6 & 70.63 & 59.85 \\
\hline Solar 7 & 0 & 4.4 \\
\hline Solar 8 & 0.02 & 40.86 \\
\hline $\begin{array}{c}\text { Total } \\
\text { capacity/kW }\end{array}$ & 682.58 & 1251.14 \\
\hline $\begin{array}{c}\text { Total cost/10 } \\
\text { yuan }\end{array}$ & 7374.4 & 7387.67 \\
\hline \begin{tabular}{c} 
Woun \\
\hline
\end{tabular} & & \\
\hline
\end{tabular}


It can be seen from the calculation results that when planning the power system, considering the complementary characteristics of wind-solar power in each time period can increase the capacity plan of wind power and photovoltaic power on the basis of basically unchanged investment costs, and enhance the competition of wind power and photovoltaic power compared with thermal power, so as to make full use of the potential of wind and solar power generation and increase the consumption of new energy.

\section{Conclusion}

This paper aims to study the joint planning method of transmission and distribution network considering the complementary characteristics of wind-solar time and space. First, a wind-solar joint distribution model based on the time-varying Copula theory is established, and a wind-solar output simulation method considering the complementary characteristics of time and space is proposed. Then, taking the system investment and new energy consumption capacity as the optimization goals, and taking the system power balance, line transmission capacity, conventional generator set output and climbing capacity as constraints, the joint planning model of transmission and distribution network is established. Finally, the analysis of calculation examples proves that the joint planning model of transmission and distribution network considering the complementary characteristics of wind-solar time and space can significantly improve the capacity of the grid to absorb new energy.

\section{Acknowledgments}

This work was financially supported by the State Grid Hubei Electric Power Company Science and Technology project(52153818001F).

\section{References}

1. Xi. Wang, Lizi. Zhang. Multi-objective grid planning for wind farms using NSGA-II hybrid intelligent algorithm $[\mathrm{J}]$. Proceedings of the CSEE, 2011, 31(19):17-24. (in Chinese)

2. Jun. Liu, Hui. Li, Ming Zhou, et al. Multi-objective power grid planning considering wind speed correlation[J]. Electric Power Automation Equipment, 2015, 35(10):87-94. (in Chinese)

3. Chengxin. Li, Zhaoyang. Dong, Guo Chen, et al. Flexible transmission expansion planning associated with large-scale wind farms integration considering demand response[J]. IET Generation, Transmission \& Distribution, 2015, 9(15):2276-2283.

4. Yue. Zhang, Xiuli. Wang, Pingliang Zeng, et al. Coordinated planning of source and network based on Copula theory considering wind power correlation [J]. Automation of Electric Power Systems, 2017, 41(09):102-108. (in Chinese)
5. Libo. Zhang, Haozhong. Cheng, Pingliang Zeng, et al. Transmission network planning based on uncertainty theory $[\mathrm{J}]$. Automation of Electric Power Systems, 2016, 40(16):159-167. (in Chinese)

6. Chang M S. A scenatio-based mixed integer linear programming model for composite power system expansion planning with greenhouse gas emission controls[J]. Clean Technologies \& Environmental policy, 2014, 16(6):1001-1014. 